\title{
Colliding molecular clouds in head-on galaxy collisions
}

\author{
J. Braine ${ }^{1}$, U. Lisenfeld ${ }^{2}$, P.-A. Duc ${ }^{3}$, E. Brinks ${ }^{4}$, V. Charmandaris ${ }^{5,6}$, and S. Leon ${ }^{2}$ \\ 1 Observatoire de Bordeaux, UMR 5804, CNRS/INSU, BP 89, 33270 Floirac, France \\ 2 Instituto de Astrofísica de Andalucía, CSIC, Apdo. Correos 3004, 18080 Granada, Spain \\ 3 CNRS URA 2052 and CEA/DSM/DAPNIA, Service d'Astrophysique, Saclay, 91191 Gif-sur-Yvette Cedex, France \\ ${ }^{4}$ INAOE, Apdo. Postal 51 y 216, Puebla, Pue. 72000, Mexico \\ 5 Cornell University, Astronomy Department, Ithaca, NY 14853, USA \\ ${ }^{6}$ Chercheur Associé, Observatoire de Paris, LERMA, 61 Av. de l’Observatoire, 75014 Paris, France
}

Received 24 November 2003 / Accepted 13 January 2004

\begin{abstract}
We present further observations of molecular gas in head-on collisions of spiral galaxies, this time of the $\mathrm{CO}(J=1 \rightarrow 0)$ and $\mathrm{CO}(J=2 \rightarrow 1)$ lines in the UGC 813 - UGC 816 system. UGC $813 / 6$ are only the second known example of head-on spiral-spiral collisions, the first example being the UGC 12914/5 pair. Strong CO emission is present in the bridge between UGC 813 and 816, unassociated with stellar emission, just as in UGC 12914/5. The CO emission from the UGC 813/6 bridge, not counting the emission from the galaxies themselves, is at least that of the entire Milky Way. Collisions of gas-rich spirals are really collisions between the interstellar media (ISMs) of the galaxies. We show that collisions between molecular clouds bring $\mathrm{H}_{2}$ into the bridge region. Although the dense clouds are ionized by the collisions, they cool and recombine very quickly and become molecular again even before the galactic disks separate. Because the clouds acquire an intermediate velocity post-collision, they are left in the bridge between the separating galaxies. The star formation efficiency appears low in the molecular clouds in the bridges. We speculate that the pre-stellar cores in the molecular clouds may expand during the cloud collisions, thus retarding future star formation. Because the ISM-ISM collisions discussed here require a very small impact parameter, they are rare among field spirals. In clusters, however, these collisions should be an important means of ejecting enriched gas from the inner parts of spirals.
\end{abstract}

Key words. galaxies: spiral - galaxies: evolution - galaxies: ISM - galaxies: interaction galaxies: individual: UGC 813 - galaxies: individual: UGC 816

\section{Introduction}

A new class of colliding galaxies has recently come to be recognized - galaxy encounters in which the neutral interstellar media (ISMs) of the systems hit each other in collisions with very small impact parameters, irrespective of the relative inclinations of the galactic planes. These systems are characterized by a bridge with strong synchrotron emission and abundant atomic hydrogen (Condon et al. 1993, hereafter C93). Two galaxy pairs have clearly undergone such a collision the UGC 12914/5 (C93; Jarrett et al. 1999) and UGC 813/6 (Condon et al. 2002, also VV 769) pairs. Contrary to initial expectations (C93), extremely strong $\mathrm{CO}$ emission was detected throughout the UGC 12914/5 bridge (Braine et al. 2003; Gao et al. 2003), showing that a large quantity of molecular gas is present as well. In fact, the $\mathrm{CO}$ emission from the UGC $12914 / 5$ bridge was found to be nearly 5 times that of the entire Milky Way. Due to the appearance of the synchrotron

Send offprint requests to: J. Braine,

e-mail: braine@obs.u-bordeaux 1.fr brightness contours and the magnetic field, the UGC 12914/5 system is also known as the "Taffy galaxies". The UGC $813 / 6$ pair shares these properties and thus is a member of the class of taffy galaxies. In this work, we show that the UGC $813 / 6$ bridge contains a lot of molecular gas, again like UGC 12914/5. The centers of UGC 813 and 816 were previously observed in CO(1-0) by Zhu et al. (1999).

It is commonly believed that molecular clouds are too small and dense to hit each other (i.e. they have a low filling factor, e.g. Jog \& Solomon 1992) or to be affected by collisions with more diffuse atomic or ionized hydrogen clouds. In addition to presenting the new results for UGC $813 / 6$, the goal of this work is to show that indeed collisions of molecular clouds are plausible and even inevitable in collisions of gas-rich galaxies. As the surface filling factor of the molecular gas in the spiral disks increases, a head-on collision of the galaxies becomes radically more efficient at drawing dense gas out of the parent disks and into the region between the two galaxies which separate after collision. The fate of the gas is unknown and probably quite dependent on whether the galaxies merge post-collision or not. 
Such collisions are, however, likely the only means of forcing much of the gas out of the inner regions of spirals because tidal forces primarily affect the less bound external regions.

The stars are not affected by collisions of the ISMs of the galaxies and of course stars do not collide with each other. However, the optical appearance can be perturbed by the tidal forces which affect both stars and gas. The slower the relative velocities of the galaxies in the collision, the stronger the effect of the tidal forces will be.

The UGC $813 / 6$ bridge system is actually part of a triplet, the third member being CGCG 551-011 about $50 \mathrm{kpc}$ away and at the systemic velocity of the UGC $813 / 6$ pair, about $5200 \mathrm{~km} \mathrm{~s}^{-1}$. Following Condon et al. (2002) we use the optical convention $(v=c z)$ for all velocities. The atomic hydrogen (HI) column densities are extremely high in the bridge region, reaching about $3 \times 10^{21} \mathrm{~cm}^{-2}$. Two $\mathrm{HI}$ peaks, $\mathrm{HI}_{\mathrm{E}}$ and $\mathrm{HI}_{\mathrm{W}}$, are also present in the outer parts of the common HI envelope and are probably tidal features. The western HI peak coincides with a blue object. The eastern HI peak has no obvious optical counterpart but is close to the optical tidal tail.

Condon et al. (2002) suggests that UGC 813 and UGC 816 are undergoing their first collision (some 40 to 50 Myrs ago) and are now separating at about $400-500 \mathrm{~km} \mathrm{~s}^{-1}$. Contrary to the UGC $12914 / 5$ system, the UGC $813 / 6$ disks are rotating in the same direction. CGCG 551-011 is also a spiral galaxy but has been stripped of most of its HI and has a strong compact radio continuum source. Without numerical simulations, we cannot determine whether the tidal features could have been generated by a collision between UGC 816 and CGCG 551-011 some 200 Myr ago, stripping CGCG 551-011 of most of its HI and feeding the central source.

After describing the observations of the UGC 813/6 system, we present the $\mathrm{CO}$ spectra and compare these with the HI data from Condon et al. (2002). Using the information available for both the UGC $12914 / 5$ and UGC $813 / 6$ pairs, we then show that molecular clouds can collide and drive large amounts of dense gas out of the spiral disks, despite earlier assumptions to the contrary. The differences with respect to previous work are explained and a general scenario proposed for how the gas, particularly the dense gas, gets into the bridge region for this type of collision. Taking $H_{0}=70 \mathrm{~km} \mathrm{~s}^{-1} \mathrm{Mpc}^{-1}$, we assume a distance to the UGC 813/6 group of $75 \mathrm{Mpc}$.

\section{Observations}

The UGC $813 / 6$ bridge, galaxy centers, and the outlying HI maxima were observed in the $\mathrm{CO}(1 \rightarrow 0)$ and $\mathrm{CO}(2 \rightarrow 1)$ to search for molecular gas. The observations were carried out with the 30 meter millimeter-wave telescope on Pico Veleta (Spain) run by the Institut de Radio Astronomie Millimétrique (IRAM) in August 2003. The $\mathrm{CO}(1 \rightarrow 0)$ and $\mathrm{CO}(2 \rightarrow 1)$ transitions at 115 and $230 \mathrm{GHz}$ respectively were observed simultaneously and in both polarizations. A bandwidth of over $1300 \mathrm{~km} \mathrm{~s}^{-1}$ was available in both transitions using the two $512 \times 1 \mathrm{MHz}$ filterbanks at $3 \mathrm{~mm}$ and the two $256 \times 4 \mathrm{MHz}$ filterbanks at $1 \mathrm{~mm}$. The band was centered around $c z=$ $5200 \mathrm{~km} \mathrm{~s}^{-1}$, where $z$ is the redshift, corresponding to redshifted frequencies of 113.3059 and $226.6074 \mathrm{GHz}$.
System temperatures were typically $200-300 \mathrm{~K}$ at $3 \mathrm{~mm}$ and twice as high for the $\mathrm{CO}(2-1)$ transition $\left(T_{A}^{*}\right.$ scale). The forward (main beam) efficiencies at Pico Veleta are currently estimated at $0.95(0.74)$ at $115 \mathrm{GHz}$ and $0.91(0.54)$ at $230 \mathrm{GHz}$. At the redshifted frequencies the half-power beamwidths are $22^{\prime \prime}$ and $11^{\prime \prime}$. All observations were done in wobbler-switching mode, usually with a throw of $100^{\prime \prime}$ but sometimes more or less depending on the position observed, in order to be sure not to have emission in the reference beam. Pointing was checked on the nearby quasar $0133+476$ every 60-80 min with pointing errors usually less than $3^{\prime \prime}$.

Data reduction was straightforward. After eliminating the few obviously bad spectra or bad channels, the spectra for each position and each transition were summed. Only zero-order baselines (i.e. continuum levels) were subtracted to obtain the final spectra. Where no CO line was obvious, the line windows were based on the HI spectra.

\section{Results}

The centers of both galaxies and 4 of the 5 bridge regions were clearly detected in both transitions. In Fig. 1 we show the $\mathrm{CO}(1-0)$ and $\mathrm{CO}(2-1)$ spectra for the galaxy centers and bridge along with the HI spectra at those positions at $16^{\prime \prime}$ resolution, intermediate between the $\mathrm{CO}(1-0)$ and $\mathrm{CO}(2-1)$ angular resolutions. The northernmost bridge position shows positive flux at the appropriate velocities. No line is obvious in $\mathrm{CO}(2-1)$ although it covers an optically brighter region (a tidal arm) than the bridge center (Fig. 2). All of the pointing centers are shown in Fig. 2. In Fig. 3 we show the $\mathrm{CO}(1-0), \mathrm{CO}(2-1)$, and $\mathrm{HI}$ spectra of the eastern and western HI peaks. No CO emission was detected despite the strong HI.

Comparison of the $\mathrm{CO}(1-0)$ observations at $22^{\prime \prime}$ (this paper) and 55" (Zhu et al. 1999) suggests that in UGC 813 the molecular gas is more centrally concentrated than in UGC 816 because in UGC 813 the line is much brighter when observed at high resolution.

In Table 1 we give the $\mathrm{CO}$ intensities for each transition and the corresponding $\mathrm{H}_{2}$ and $\mathrm{HI}$ column densities for each of the positions observed. While the $\mathrm{CO}$ emission from the galaxies is stronger than in the bridge, the $\mathrm{CO}$ emission from the bridge is at least that of the Milky Way. For a $\mathrm{CO}-\mathrm{H}_{2}$ conversion factor of $N\left(\mathrm{H}_{2}\right) / I_{\mathrm{CO}}=2 \times 10^{20}$ molecules $\mathrm{cm}^{-2}\left(\mathrm{~K} \mathrm{~km} \mathrm{~s}^{-1}\right)^{-1}$ (e.g. Dickman et al. 1986), the bridge is about half molecular and half atomic gas - some $2 \times 10^{9} M_{\odot}$ of each. This is based on the central bridge position alone. For the other positions, it is difficult to separate the emission from the disks from that of the bridge, such that the above represents a lower limit to the true bridge $\mathrm{CO}$ emission.

As can be readily seen from Fig. 1, the molecular and atomic gas line profiles are very similar. Furthermore, assuming the $N\left(\mathrm{H}_{2}\right) / I_{\mathrm{CO}}$ factor used is appropriate, the hydrogen column densities are also similar in the bridge region (the HI spectra in Fig. 1 are scaled so that the same line area as in CO represents the same $\mathrm{H}$-atom column density). The molecular gas dominates in the galactic centers and in the densest parts of the bridge. This is obviously not true of tidal tails, which preferentially bring material out of the $\mathrm{HI}$ - dominated outer regions of 


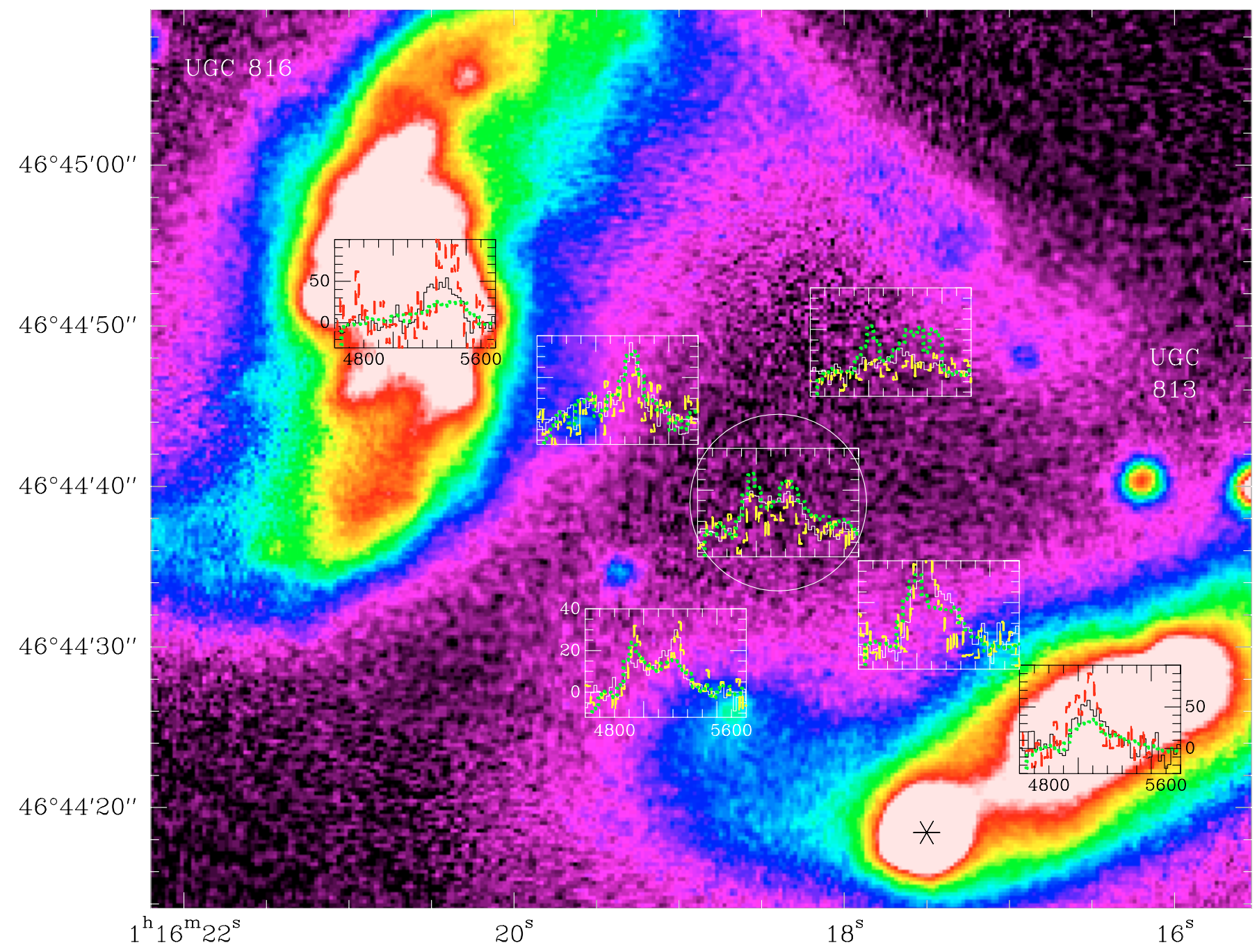

Fig. 1. $\mathrm{CO}(1 \rightarrow 0), \mathrm{CO}(2 \rightarrow 1)$, and $\mathrm{HI}$ spectra at the positions observed in the UGC 813/6 galaxies and bridge, using respectively black solid (white solid in the bridge), red dashed (yellow dashed in the bridge), and green dotted lines. The circle indicates the size of the CO(2-1) beam. UGC 813 is the edge-on spiral to the lower right and UGC 816 is to the left, less inclined and at a higher velocity. The asterisk marks a bright foreground star. For the CO spectra, the intensity scale is main beam antenna temperature in $\mathrm{mK}$ ( -30 to $100 \mathrm{mK}$ for the galaxy centers and -12 to $40 \mathrm{mK}$ for the bridge positions). The $\mathrm{HI}$ spectra are plotted such that equal $\mathrm{CO}(1 \rightarrow 0)$ and $\mathrm{HI}$ intensity correspond to equal $\mathrm{H}$-atom column densities in $\mathrm{H}_{2}$ and in $\mathrm{HI}$. It is thus apparent that in the bridge, the $\mathrm{HI}$ and $\mathrm{H}_{2}$ column densities are similar, assuming the $N\left(\mathrm{H}_{2}\right) / I_{\mathrm{CO}}$ factor is correct. The underlying image is an $R$ band image taken with the Instituto de Astrofísica de Andalucía (IAA) $1.5 \mathrm{~m}$ telescope on Pico Veleta.

spirals. The CO line intensities given in Table 1 for the bridge positions are unheard of for tidal material. The two HI peaks, at about 1-2 arcmin from the bridge and marked as $\mathrm{HI}_{\mathrm{E}}$ and $\mathrm{HI}_{\mathrm{W}}$, appear to be at the ends of tidal arms or tails and indeed the $\mathrm{HI}$ emission is strong but no $\mathrm{CO}$ has been detected there so far (Fig. 3). The $\mathrm{H}_{2} / \mathrm{HI}$ mass ratio in the tidal material is less than $10 \%$, compatible with the less evolved Tidal Dwarf Galaxies (TDG) for which $\mathrm{CO}$ detections have been obtained (Braine et al. 2001). Figure 4 presents an $\mathrm{H} \alpha$ image of the UGC $813 / 6$ system with HI contours superposed. No star formation is observed near the northeastern $\mathrm{HI}$ peak $\left(\mathrm{HI}_{\mathrm{E}}\right)$, which is clearly not, or at least not yet, a TDG.

To further distinguish between bridge and tidal features, we plot the synchrotron emission (Condon et al. 2002) over the
$R$ band image in Fig. 5. Just as in the Taffy galaxies, the $\mathrm{CO}$ emission follows the synchrotron more than any tidal features. Strong extra-disk synchrotron emission is one of the characterizing features of ISM-ISM collisions and not of tidal arms or tails.

In the Taffy galaxies (UGC 12914/5), the CO/HI ratio in the bridge is higher. The $\mathrm{CO}$ and HI line profiles in the UGC $12914 / 5$ bridge are quite different; this is, however, probably a projection effect because we see the HI from UGC 12914 as if it were in the bridge while it is actually far behind (see Braine et al. 2003). UGC 12914 and 12915 are generally bigger and more gas-rich than the UGC $813 / 6$ system. As we will see, this is important for the amount of molecular gas that can be stripped through cloud-cloud collisions. 


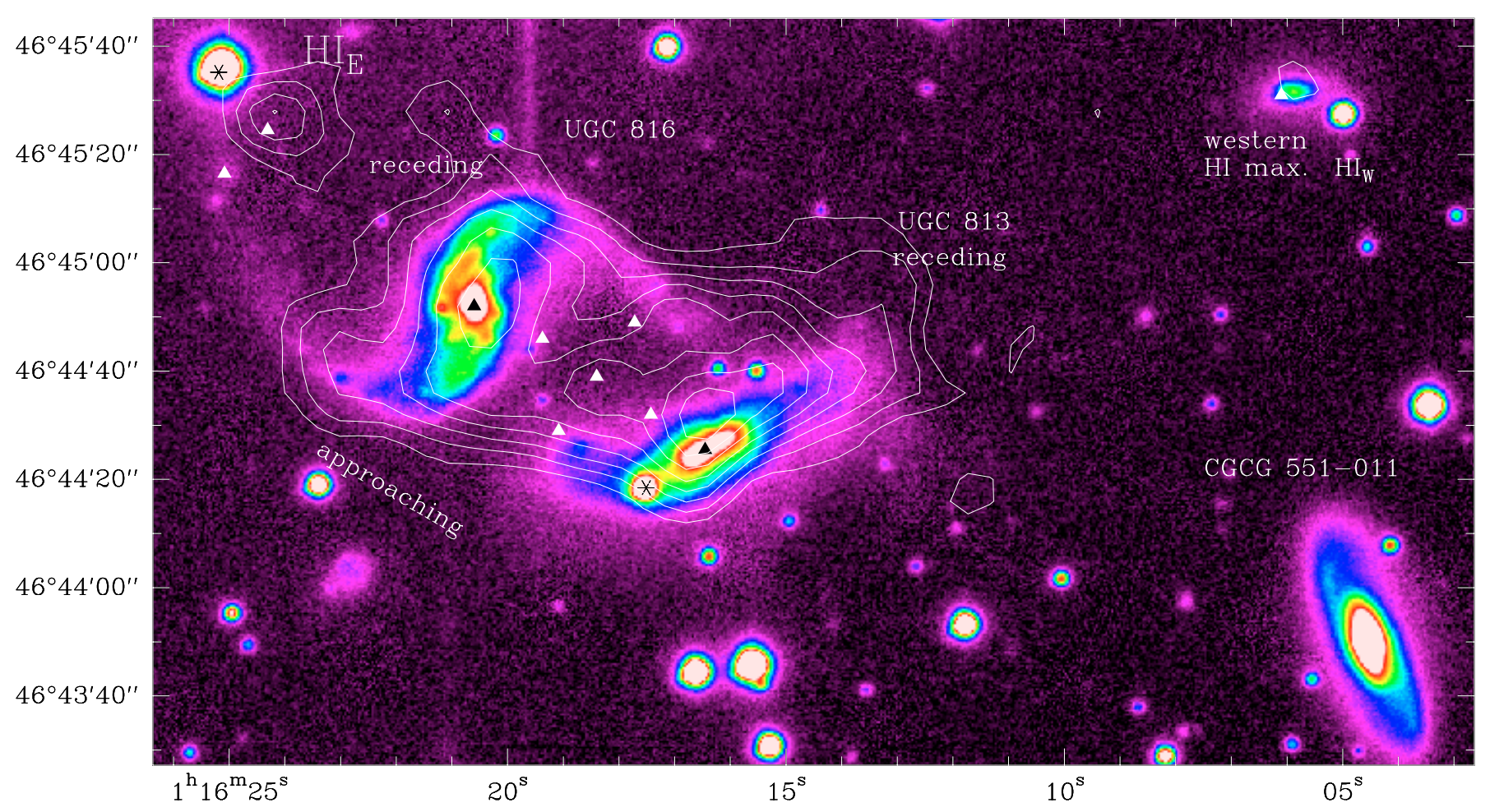

Fig. 2. $R$ band image taken with the IAA $1.5 \mathrm{~m}$ telescope of the entire UGC 813/6 system with HI column density contours from Condon et al. (2002). The centers of UGC 813 and 816 are marked by black triangles. The bridge positions observed are the five white triangles between the galaxies. These are the positions whose spectra are shown in Fig. 1. The triangles to the extreme West and East, slightly north of UGC 816, mark the positions of the Hi peaks whose spectra are shown in Fig. 3. Asterisks mark the positions of some galactic stars which could be confused with other features. The spiral galaxy seen nearly edge-on in the lower right is CGCG 551-011.

Table 1. Complete list of positions observed in CO, corresponding to the triangles in Fig. 2. The offsets are in arseconds with respect to the $(0,0)$ position of the source. Columns 3 and 4 give the $\mathrm{CO}(1-0)$ and $\mathrm{CO}(2-1)$ integrated intensities and Cols. 5 and 6 the $\mathrm{H}_{2}$ and $\mathrm{HI}$ column densities. The $\mathrm{CO}$ line window for the "HI $\mathrm{I}_{\mathrm{E}}$ " positions is 5370 to $5470 \mathrm{~km} \mathrm{~s}^{-1}$ and 5170 to $5270 \mathrm{~km} \mathrm{~s}^{-1}$ for the "HI⿰" the HI spectra shown in Fig. 3. Uncertainties are $1 \sigma$ based on the rms noise and the line width. Upper limits to $N\left(\mathrm{H}_{2}\right)$ are $3 \sigma$.

\begin{tabular}{lcccccrc}
\hline \hline Source & $\begin{array}{c}\text { RA } \\
(\mathrm{J} 2000)\end{array}$ & $\begin{array}{c}\text { Dec } \\
(\mathrm{J} 2000)\end{array}$ & $\begin{array}{c}\text { Offset } \\
\operatorname{arcsec}\end{array}$ & $\begin{array}{c}I_{\mathrm{CO}(1-0)} \\
\mathrm{K} \mathrm{km} \mathrm{s}^{-1}\end{array}$ & $\begin{array}{c}I_{\mathrm{CO}(2-1)} \\
\mathrm{K} \mathrm{km} \mathrm{s}^{-1}\end{array}$ & $\begin{array}{c}N\left(\mathrm{H}_{2}\right) \\
10^{20} \mathrm{~cm}^{-2}\end{array}$ & $\begin{array}{c}N(\mathrm{HI}) \\
10^{20} \mathrm{~cm}^{-2}\end{array}$ \\
\hline UGC 813 & $01: 16: 16.45$ & $46: 44: 25$ & $(0,0)$ & $12.2 \pm 0.9$ & $10.3 \pm 1.3$ & 24 & 38 \\
UGC 816 & $01: 16: 20.6$ & $46: 44: 52$ & $(0,0)$ & $11.1 \pm 0.6$ & $13.9 \pm 2.1$ & 22 & 37 \\
Bridge & $01: 16: 18.4$ & $46: 44: 39$ & $(0,0)$ & $7.0 \pm 0.2$ & $3.7 \pm 0.4$ & 14 & 35 \\
Bridge & & & $(7,-10)$ & $6.6 \pm 0.4$ & $7.4 \pm 0.5$ & 13 & 27 \\
Bridge & & & $(-7,10)$ & $2.5 \pm 0.3$ & $0.7 \pm 0.4$ & 5 & 32 \\
Bridge & & & $(10,7)$ & $6.7 \pm 0.4$ & $4.5 \pm 0.7$ & 13 & 30 \\
Bridge & & & $(-10,-7)$ & $10.5 \pm 0.5$ & $8.3 \pm 0.6$ & 21 & 35 \\
HI $_{\mathrm{E}}$ & $01: 16: 24.31$ & $46: 45: 24$ & $(0,0)$ & $0 \pm 0.14$ & $0 \pm 0.4$ & $\lesssim 1$ & 22 \\
HI $_{\mathrm{E}}$ & & & $(8,-8)$ & $0 \pm 0.10$ & $0 \pm 0.2$ & $\lessgtr 0.6$ & 8 \\
$\mathrm{HI}_{\mathrm{W}}$ & $01: 16: 06.1$ & $46: 45: 31$ & $(0,0)$ & $0 \pm 0.08$ & $0 \pm 0.2$ & $\lesssim 0.5$ & 10 \\
\hline
\end{tabular}

\section{Why is $\mathrm{H}_{2}$ found in the bridges?}

In the following we will address the question of the origin of the $\mathrm{H}_{2}$ found in the bridge between UGC 813 and UGC 816 . As this galaxy pair is very similar to the Taffy galaxies UGC 12914/5 and we also found abundant molecular gas there, we will attempt to provide a more general explanation which applies to any Taffy-type interaction. We will assume that the $N\left(\mathrm{H}_{2}\right) / I_{\mathrm{CO}}$ factor adopted here is valid for the bridge molecular gas. Gao et al. (2003) adopt a higher $N\left(\mathrm{H}_{2}\right) / I_{\mathrm{CO}}$ factor in their work on UGC 12914/5; Braine et al. (2003) discuss why the factor they adopt is probably too high. The first question is perhaps whether the molecular gas could form from the atomic gas in the bridge.

\subsection{Could the $\mathrm{H}_{2}$ form out of the $H_{l}$ in the bridge?}

The HI column density is typically $2-4 \times 10^{21} \mathrm{~cm}^{-2}$ in the bridges. If the depth of the bridge is similar to its extent 


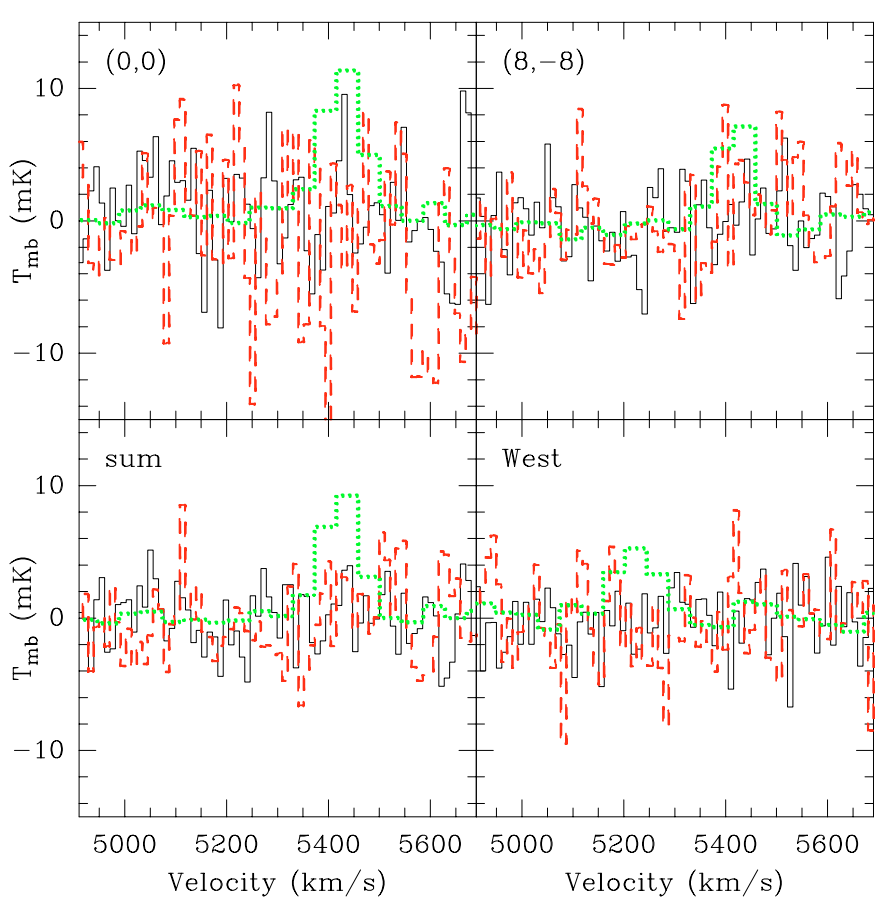

Fig. 3. $\mathrm{CO}(1-0), \mathrm{CO}(2-1)$ and HI spectra of the "TDG candidate" positions. The brightness scale is for the $\mathrm{CO}(1-0)$ line and should be doubled for the $\mathrm{CO}(2-1)$ line (i.e. the $\mathrm{CO}(2-1)$ spectra have been divided by two). The HI scale has been divided by 4 such that if the column density of molecular gas were $25 \%$ of the atomic gas column density, then the spectra would be of the same height. It is immediately obvious that the $\mathrm{CO}$ intensities indicate low $\mathrm{H}_{2}$ column densities compared to HI. The lower left panel shows the sum of the two eastern positions shown in the top two panels. The positions are shown in Fig. 2 where they are indicated by triangles.

perpendicular to the collision direction, then the neutral gas is spread over some $10-20 \mathrm{kpc}$. The average volume density is then $\lesssim 0.1$. Braine et al. (2001) estimate that $10^{7} / n$ years (i.e. $\gtrsim 10^{8}$ yrs in the bridges) is necessary to convert $20 \%$ of the $\mathrm{HI}$ into $\mathrm{H}_{2}$. Once some of the $\mathrm{HI}$ has become $\mathrm{H}_{2}$ the process becomes less efficient. While local density enhancements could allow some $\mathrm{H}_{2}$ formation from the atomic gas, it is unlikely that the large quantities of $\mathrm{H}_{2}$ observed could have formed from $\mathrm{HI} . \mathrm{H}_{2}$ is in fact the dominant phase in the Taffy bridge and is roughly half of the UGC $813 / 6$ bridge ISM. If the grains are warm then the ability of $\mathrm{H}$-atoms to form $\mathrm{H}_{2}$ on the grain surfaces is greatly reduced (Hollenbach \& Salpeter 1971). Furthermore, the efficiency of $\mathrm{HI}$ to $\mathrm{H}_{2}$ conversion is obviously reduced if grains are destroyed in the strong shocks generated in cloud collisions (whether in atomic or molecular clouds).

There is a further reason for why clouds which are not initially dense will not be able to contract. In both systems, and particularly the Taffy galaxies, the spirals are massive (several $10^{11} M_{\odot}$ each from C93 and Condon et al. 2002). As a result, the tidal forces they exert on clouds are stronger than in the
Milky Way. Following Bania et al. (1986), for clouds to resist against tidal shear, their density must be $n \gtrsim 200\left(\frac{3 \mathrm{kpc}}{R}\right)^{2}$ nucleons $\mathrm{cm}^{-3}$

where $R$ is the distance from the cloud to the galactic centers. Being able to resist tidal forces is not sufficient for cloud survival but is certainly required. Thus, clouds which are not sufficiently dense after collision to form $\mathrm{H}_{2}$ rapidly may not be dense enough to survive to form $\mathrm{H}_{2}$ subsequently in the bridge.

\subsection{GMC collisions as part of galaxy collisions}

The observations show that when spiral galaxies physically collide, with the inner parts of their disks passing through each other, more than $10^{9} M_{\odot}$ of molecular gas is found in the resulting bridge region between the two galaxies. While tidal forces can drive gas out of spiral galaxies, they act on stars in the same way, such that it is difficult to imagine how a bridge with so much gas but no stars could have been formed by tidal forces alone. It has commonly been accepted (e.g. Jog \& Solomon 1992) that Giant Molecular Clouds (GMCs) do not collide whereas the more diffuse clouds of atomic gas do. The goal of this section is to demonstrate that in gas-rich but not exceptional spiral galaxies GMC-GMC collisions occur and are likely the source of the large quantities $\left(1-10 \times 10^{9} M_{\odot}\right)$ of molecular gas present in the bridges.

In the calculations we will suppose that molecular clouds are spherical and obey the so-called Larson (1981) relation with an average column density of $N\left(\mathrm{H}_{2}\right)=10^{22} \mathrm{~cm}^{-2}$. This is typical and corresponds exactly to what Jog \& Solomon (1992) assume. We define the following quantities:

- $A_{\mathrm{cl}}$, the projected cloud area, equal to $\pi R_{\mathrm{cl}}^{2}$ for identical spherical clouds where $R_{\mathrm{cl}}$ is the cloud radius;

- $n_{\mathrm{cl}}$, the number density of such clouds;

- $M_{\mathrm{cl}}$, the mass of a cloud of radius $R_{\mathrm{cl}}$;

$-z_{\mathrm{H}_{2}}$, the half thickness of the layer of molecular clouds;

- $\Sigma_{\mathrm{H}_{2}}$, the face-on $\mathrm{H}_{2}$ surface density of the spiral galaxies, expressed in $M_{\odot} \mathrm{pc}^{-2}$, typically averaged over a telescope beam;

- $\Sigma_{\mathrm{cl}}$, the $\mathrm{H}_{2}$ surface density of a cloud which is simply $160 M_{\odot} \mathrm{pc}^{-2}$ for $N\left(\mathrm{H}_{2}\right)=10^{22} \mathrm{~cm}^{-2}$;

- and the velocities $V_{\perp}$, taken to be the encounter speed perpendicular to the galactic planes and $V_{\|}$, the relative cloud velocities in the planes (which are assumed parallel as in the Taffy galaxies). The link to extragalactic observables is provided by the integrated $\mathrm{CO}(1 \rightarrow 0)$ line intensity $I_{\mathrm{CO}}$, expressed in $\mathrm{K} \mathrm{km} \mathrm{s}^{-1}$, via the conversion factor taken to be $N\left(\mathrm{H}_{2}\right) / I_{\mathrm{CO}}=2 \times 10^{20} \mathrm{H}_{2} \mathrm{~cm}^{-2}$ per $\mathrm{K} \mathrm{km} \mathrm{s}^{-1}$.

The mean free path $\lambda_{\mathrm{mfp}}$ of a spherical particle is $\lambda_{\mathrm{mfp}}=$ $1 /\left(4 A_{\mathrm{cl}} n_{\mathrm{cl}}\right)$. For a gas of particles in random motion, the mean free path is actually $\sqrt{2}$ shorter. The factor 4 comes from the fact that clouds collide if they pass within $2 R_{\mathrm{cl}}$ of each other. The number density of clouds is

$n_{\mathrm{cl}}=\frac{\Sigma_{\mathrm{H}_{2}}}{2 z_{\mathrm{H}_{2}}} \times \frac{1}{M_{\mathrm{cl}}}$, where $M_{\mathrm{cl}}=\Sigma_{\mathrm{cl}} \times A_{\mathrm{cl}}$ 
such that the mean free path is simply

$\lambda_{\mathrm{mfp}}=\frac{2 z_{\mathrm{H}_{2}} \Sigma_{\mathrm{cl}}}{4 \Sigma_{\mathrm{H}_{2}}}$

such that when $\Sigma_{\mathrm{H}_{2}}=\Sigma_{\mathrm{cl}}$, the mean free path of a cloud is $1 / 4$ of the thickness of the molecular cloud layer $2 z_{\mathrm{H}_{2}}$. This is in some ways a trivial result because $\Sigma_{\mathrm{H}_{2}}=\Sigma_{\mathrm{cl}}$ means that the galactic planes are completely covered by a layer of molecular clouds so it seems obvious that they should collide when the disks pass through each other. CO observations show that many spirals have disk CO intensities of order $I_{\mathrm{CO}} \gtrsim 20 \mathrm{~K} \mathrm{~km} \mathrm{~s}^{-1}$, corresponding to $\Sigma_{\mathrm{H}_{2}} \gtrsim 65 M_{\odot} \mathrm{pc}^{-2}$, such that the mean free path is less than the thickness of the molecular cloud layer.

The mean free path formula above gives collisions even when only parts of the spherical clouds overlap. However, when there is a transverse velocity, such as in the Taffy galaxies where the counter-rotation yields high encounter speeds parallel to the galactic planes, then the path of a cloud in the molecular layer of the other system is increased from $2 z_{\mathrm{H}_{2}}$ to $2 z_{\mathrm{H}_{2}} \sqrt{V_{\|}^{2}+V_{\perp}^{2}} / V_{\perp}$. When the parallel and perpendicular velocities are roughly equal, this lengthens the path and increases the probability of cloud collisions by $\sqrt{2}$. While the magnetic field energy in a cloud is greatly less than the kinetic energy of the collision, magnetic fields are nonetheless quite strong in dense gas, with the field strength increasing as $\sqrt{\text { density, }}$ reaching about $100 \mu \mathrm{G}$ (Crutcher 1999). As such, they could be expected to increase the effective cloud cross-section somewhat. If the galactic planes are not parallel and/or if the collision is not face-on then the cloud paths within the galactic planes are increased.

The above discussion only considers collisions between molecular clouds, showing that rather than being rare, GMC-GMC collisions on large scales are inevitable in collisions of gas-rich spirals. After (inelastic) collision, the resulting cloud and possibly cloud fragments will have a velocity intermediate between the velocities of the galactic disks. They are thus left behind in the bridge region between the separating galaxies. Whether or not the clouds fall back onto one of the galaxies depends on their velocity with respect to the galaxies and thus the collision velocity.

\subsection{Why previous results were different}

We choose to compare here with the work of Jog \& Solomon (1992) who explored this question and have been cited for why GMCs should not collide. Furthermore, the cloud properties they assume are in accord with our hypotheses - the only significant number, the average $\mathrm{H}_{2}$ column density (or projected $\mathrm{H}_{2}$ surface mass density) is the same as our adopted value. Jog \& Solomon (1992) define the mean free path by introducing a volume filling factor for molecular clouds, which is the cloud volume multiplied by the number of clouds per unit volume in the molecular layer of a spiral galaxy. Hence

$$
\begin{aligned}
f & =4 \pi R_{\mathrm{cl}}^{3} n_{\mathrm{cl}} / 3 \\
& =\left(4 \pi R_{\mathrm{cl}}^{3} / 3\right) \times \Sigma_{\mathrm{H}_{2}} /\left(2 z_{\mathrm{H}_{2}} \Sigma_{\mathrm{cl}} A_{\mathrm{cl}}\right) \\
& =\left(4 R_{\mathrm{cl}} / 3\right) \times \Sigma_{\mathrm{H}_{2}} /\left(2 z_{\mathrm{H}_{2}} \Sigma_{\mathrm{cl}}\right)
\end{aligned}
$$

after making the substitutions using the formulae above for identical spherical clouds (the hypothesis adopted by Jog \& Solomon 1992). Their mean free path is then

$\lambda_{\mathrm{mfp}}=2 R_{\mathrm{cl}} / 3 f=2 R_{\mathrm{cl}} / 3 \times 1 /(4 \pi / 3) R_{\mathrm{cl}}^{3} n_{\mathrm{cl}}=1 / 2 A_{\mathrm{cl}} n_{\mathrm{cl}}$

which is twice the value we use. This may be to ensure that only collisions with comparable molecular column densities are included, such that grazing encounters are entirely excluded. This is not the origin of the discrepancy.

Jog \& Solomon (1992) specify the volume filling factor $f$ to be $f_{\mathrm{GMC}}=0.01$ which we now express in terms closer to observables. Using the above definitions,

$$
\begin{aligned}
\Sigma_{\mathrm{H}_{2}} & =f \times 2 z_{\mathrm{H}_{2}} \Sigma_{\mathrm{cl}} A_{\mathrm{cl}} \times 3 /\left(4 \pi R_{\mathrm{cl}}^{3}\right) \\
& \approx \frac{f}{0.01} \times \frac{2 z_{\mathrm{H}_{2}}}{130 \mathrm{pc}} \times \frac{\Sigma_{\mathrm{cl}}}{R_{\mathrm{cl}}} M_{\odot} \mathrm{pc}^{-2} \\
& \approx 6 M_{\odot} \mathrm{pc}^{-2}
\end{aligned}
$$

for $2 z_{\mathrm{H}_{2}}=130 \mathrm{pc}, \Sigma_{\mathrm{cl}}=160 M_{\odot} \mathrm{pc}^{-2}$, and $R_{\mathrm{cl}}=25 \mathrm{pc}$, as in Jog \& Solomon (1992). $6 M_{\odot} \mathrm{pc}^{-2}$ corresponds to $I_{\mathrm{CO}}=$ $2 \mathrm{~K} \mathrm{~km} \mathrm{~s}^{-1}$, which is weak for the inner parts of a spiral galaxy. This is the origin of the discrepancy: the Jog \& Solomon (1992) calculations assume a very low molecular gas surface density. The value they assume is roughly the molecular gas surface density of the Milky Way averaged over the entire optical disk. In the molecular ring of our Galaxy the $\mathrm{H}_{2}$ surface density is much higher. This is why grazing encounters should not be efficient at generating GMC-GMC collisions but collisions of the inner parts of spirals will be.

\section{The collision scenario}

Unfortunately, no simulations of colliding neutral clouds, especially with magnetic fields, are available. Harwit et al. (1987) explore analytically what happens when two (big) disks of dense gas collide - we largely follow their scenario. The crossing or collision time of two clouds is

$t=D_{\text {eff }} / V=10^{5} \frac{\sqrt{A_{\mathrm{cl}}}}{50 \mathrm{pc}} \times \frac{500 \mathrm{~km} \mathrm{~s}^{-1}}{V}$ years.

As two clouds collide, whether originally atomic or molecular, the colliding parts are instantly ionized and reach temperatures of $T \gtrsim 10^{6} \mathrm{~K}$ (depending on original collision velocity). As the two clouds continue to move into each other, the central region will be compressed and will cool very rapidly. A similar scenario is proposed in the case of interstellar shocks in Fig. 1 of Hollenbach \& McKee (1989). The pressure in the central region is very high so the gas will spread out perpendicular to the collision direction, as in the Mair et al. (1988) simulations. How much gas flows to the side is highly dependent on the geometry of the clouds and of the collision. With the exception of gas flow towards the exterior, the collisions are concisely described by Fig. 4 and Sect. 8 of Harwit et al. (1987). The Struck (1997) simulations also suggest that ISM-ISM collisions can bring large amounts of gas into the bridge and that the gas is dense enough to cool, although he does not go into the details of cooling or the cloud collisions themselves. 


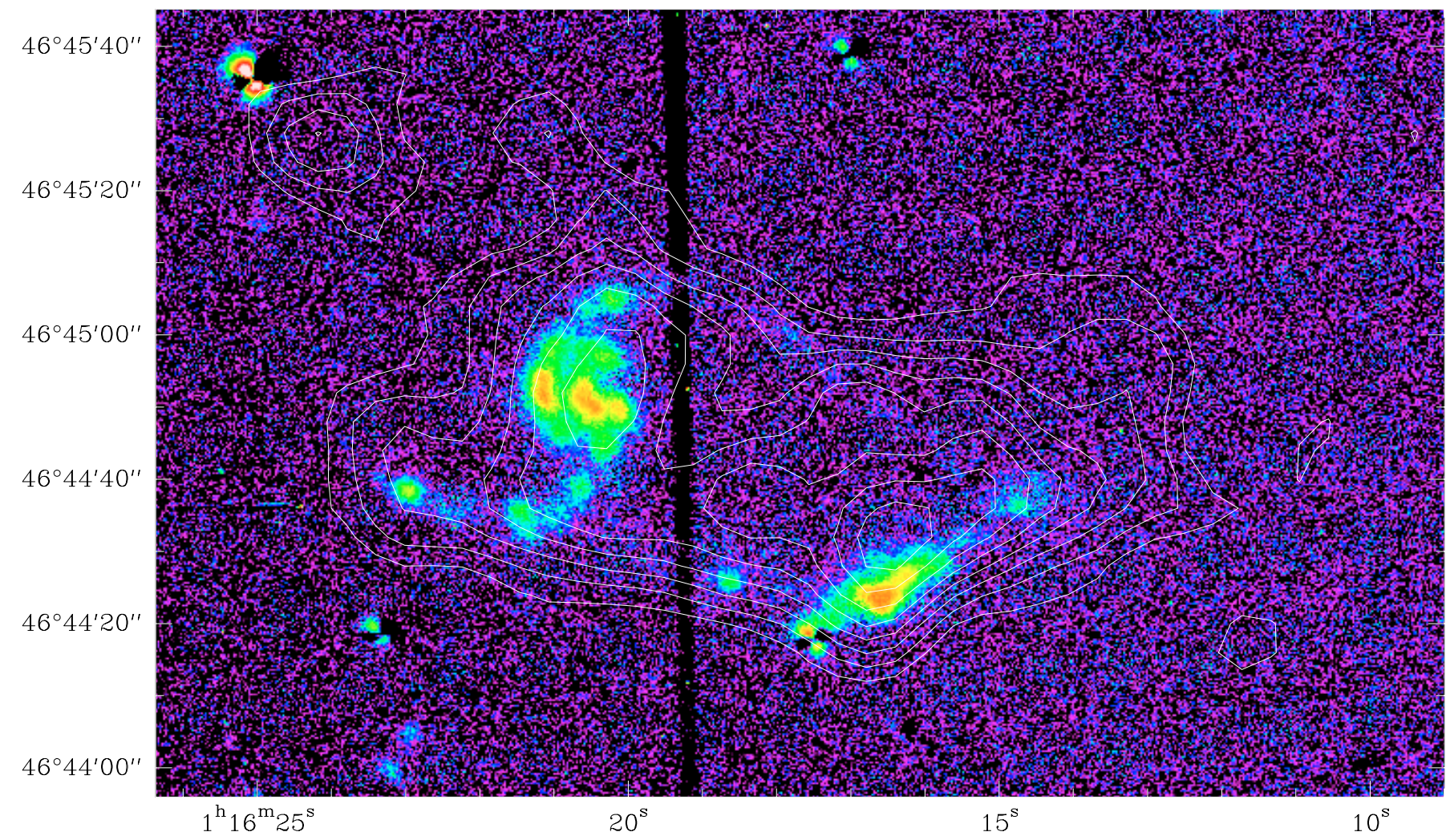

Fig. 4. $\mathrm{H} \alpha$ image after broadband continuum subtraction taken with the San Pedro Mártir, Baja California (Mexico), $2.12 \mathrm{~m}$ telescope with the same HI column density contours as in Fig. 2. The dark vertical stripe through the bridge is due to the bright star above UGC 816 . A fairly bright HII region is seen in the southern part of the bridge. No H $\alpha$ emission is observed near the NE HI peak.

The hot gas $\left(10^{6} \lesssim T \lesssim 10^{7} \mathrm{~K}\right)$ cools very rapidly (Gaetz \& Salpeter 1983; Harwit et al. 1987) down to below $10^{4} \mathrm{~K}$. The cooling timescale, only a few years for molecular cloud collisions, is much shorter than the collision timescale. Thus, the material at the barycenter of the collision will very quickly become cool and neutral, provided it is sufficiently dense. Hollenbach \& McKee (1989) estimate the column density necessary to form $\mathrm{H}_{2}$ to be $N_{1 / 2} \approx 4 \times 10^{21} \mathrm{~cm}^{-2}$ where the subscript $1 / 2$ indicates changing half of the $\mathrm{HI}$ into $\mathrm{H}_{2}$. The longest time scale is also the $\mathrm{H}_{2}$ formation time scale. All of the time scales vary as the inverse of the density so collisions of dense material have the best chances of producing predominantly cool molecular gas at the end of the collision. This means that the molecular gas we observe in the bridge region left the galactic disks in molecular form.

Most collisions will obviously not be head-on collisions between identical clouds. Without detailed simulations of neutral cloud collisions, however, it is difficult to estimate, for each geometry and possible cloud type (atomic, molecular), the fraction of the gas post-collision which will be in atomic, molecular, or ionized form. It is likely that head-on collisions of large atomic clouds will also produce (after ionization and cooling) a high fraction of $\mathrm{H}_{2}$. Given that (i) collisions between molecular clouds are not only possible but inevitable on large scales once the surface filling factor $\left(\sim \Sigma_{\mathrm{H}_{2}} / \Sigma_{\mathrm{cl}}\right)$ of the colliding spirals is greater than about $0.2\left(\lambda_{\mathrm{mfp}} \sim\right.$ thickness of molecular gas layer $)$ and (ii) that collisions between dense clouds are most likely to generate cool dense (molecular) gas post-collision, it is reasonable to expect much of the $\mathrm{H}_{2}$ in the bridge regions to be due to collisions of molecular clouds.

Observationally, if $\mathrm{HI}$ - HI cloud collisions were efficient in creating $\mathrm{H}_{2}$, then the $\mathrm{HI} / \mathrm{H}_{2}$ ratio in the Taffy bridge would be relatively constant. However, the $\mathrm{H}_{2}$ in the bridge decreases rather sharply from the center outward, just like the centrally concentrated $\mathrm{CO}$ emission in spirals, and the $\mathrm{HI}$ is extended both in the bridge and in the spirals. The most likely explanation is that molecular cloud collisions generate much of the observed molecular gas in the bridges.

\section{Star formation in the bridges?}

With such large quantities of molecular gas present, star formation rates of $1-10 M_{\odot}$ per year are expected in the bridges, if the gas is in a "normal" state, capable of forming stars as in spirals. From the $\mathrm{H} \alpha$ and $\mathrm{CO}$ emission of spirals, a star formation rate (SFR) of about $1 M_{\odot}$ per year is estimated per $10^{9} M_{\odot}$ of molecular gas (Kennicutt 1998; Braine et al. 2001, Sect. 3). The bridges are estimated to be about 20-30 Myr and 40-50 Myr old for the UGC 12914/5 (Taffy) and UGC 813/6 systems (Condon et al. 1993, 2002). Such times are well above the collapse times for cloud cores.

From the CO emission from the bridges, the SFRs expected in the UGC 12914/5 and UGC 813/6 bridges are about 9 and $2 M_{\odot} \mathrm{yr}^{-1}$. Jarrett et al. (1999) find that with the 


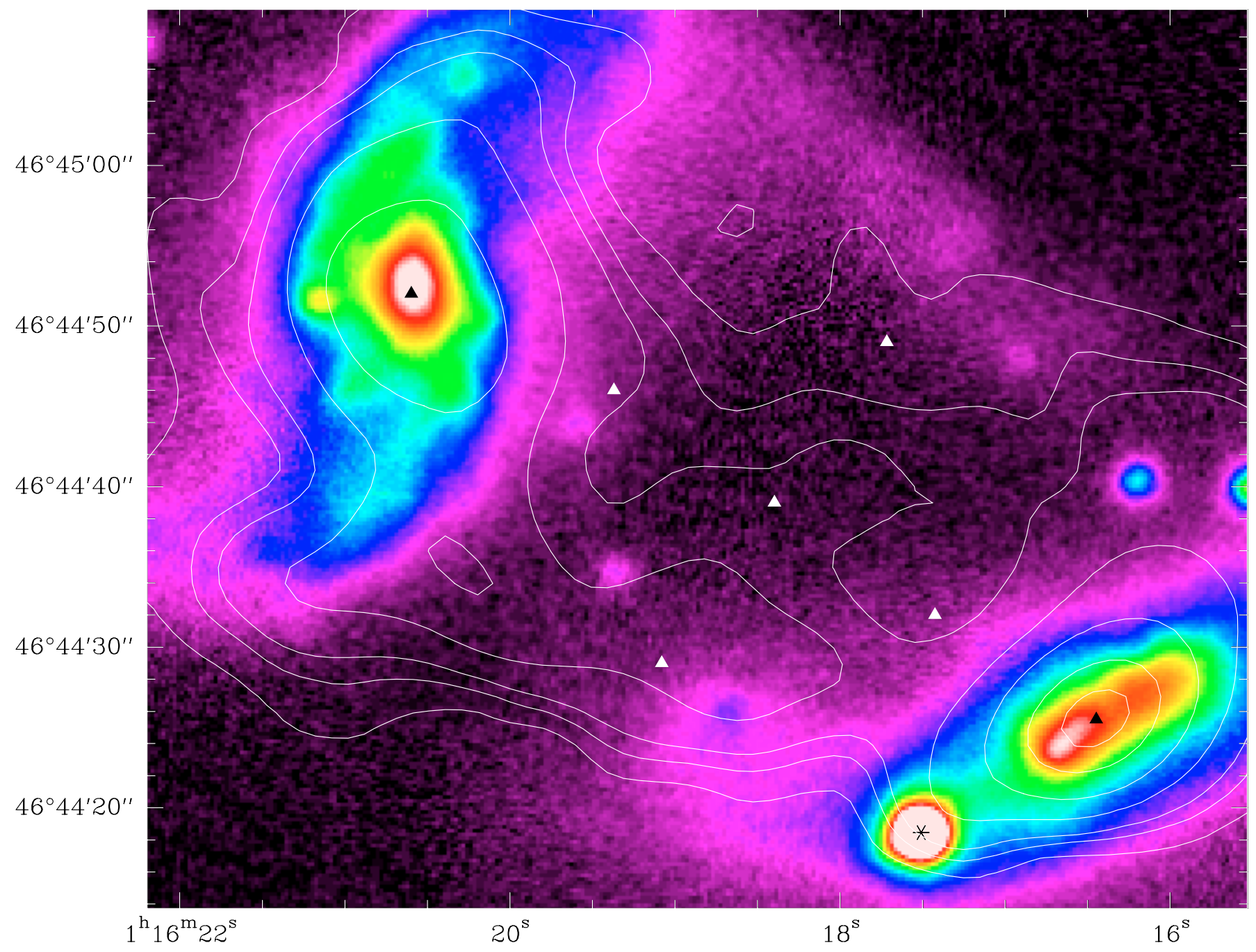

Fig. 5. $R$ band image with $1.4 \mathrm{GHz}$ synchotron emission contours superposed. Triangles indicate the positions observed in $\mathrm{CO}$ and the asterisk a star. While the optical emission in the NW bridge position (near the northern tidal arm) is stronger than in the others, the CO emission is much weaker there.

exception of the single giant HII region near UGC 12915, the UGC $12914 / 5$ bridge is devoid of stars and star formation. From the slit spectrum of the UGC 12914/5 bridge presented by Braine et al. (2003), there is no sign of star formation (except in the HII region) although the molecular gas surface density is nearly constant (Braine et al. 2003; Gao et al. 2003) throughout the bridge. A single discrete HII region is observed in the UGC $813 / 6$ bridge (Fig. 4). The luminosity expected from bridges with several $M_{\odot} \mathrm{yr}^{-1}$ of star formation is not observed. Because any stars formed in the bridge are necessarily quite young, could they still be dust-enshrouded, such that we would not see their light?

From the $\mathrm{H} \alpha$ observations (Bushouse 1987; Bushouse \& Werner 1990) of the Taffy pair, we estimate that the SFR is about $0.1-0.3 M_{\odot} \mathrm{yr}^{-1}$. From the Far-IR (IRAS) luminosity of the pair, about $4 \times 10^{10} L_{\odot}$ between 42 and $122 \mu \mathrm{m}$, the SFR is between 5 and $10 M_{\odot} \mathrm{yr}^{-1}$, more than half of which is in UGC 12915. In the Taffy galaxies there is a lack of FIR emission in the bridge (Zink et al. 2000) but dust, perhaps only small grains, is detected via its Mid-IR emission. The flux in the bridge at $15 \mu \mathrm{m}$ measured by Jarrett et al. (1999) with ISOCAM is about $5 \%$ of that measured in each of the galaxies themselves. This is in rough agreement with the $\mathrm{H} \alpha$ based SFR so we estimate that the bridge SFR is between 0.1 and $0.4 M_{\odot} \mathrm{yr}^{-1}$. For the Taffy system, this is less than $10 \%$ of what is expected based on the CO luminosity.

The cloud collisions and associated shocks are expected to destroy dust grains to a large extent. No observations relevant to this are available for the UGC $813 / 6$ system. The small amount of dust in the Taffy bridge (Zink et al. 2000) means that it is unlikely that star formation is hidden through extinction by dust. The dip in the FIR emission in the bridge and the low SFR calculated from the MIR emission indicates that less star formation is occurring in the Taffy bridge than would be expected given the strong $\mathrm{CO}$ emission. As discussed in Braine et al. (2003), the $N\left(\mathrm{H}_{2}\right) / I_{\mathrm{CO}}$ ratio could be lower by a factor of a few. This would reduce the amount of "missing" star formation but 
even allowing for the uncertainty in the $N\left(\mathrm{H}_{2}\right) / I_{\mathrm{CO}}$ conversion, the SFR still appears low.

In the UGC $813 / 6$ system, an HII region is present in the bridge but contributes less than $2 \%$ of the $\mathrm{H} \alpha$ luminosity of the system. The $\mathrm{H} \alpha$ emission surely suffers from extinction in the galaxies, particularly in UGC 813 which is seen edge-on. Although the pair has not been completely mapped in $\mathrm{CO}$, we estimate that the bridge contributes between 12 and $30 \%$ of the global CO luminosity. Thus, again, the SFR is only some $10 \%$ of what would be expected in "normal" molecular gas. Our tentative conclusion is that star formation in the bridge material has been retarded or even suppressed by the recent collision.

In galaxies, stars form through the collapse of dense "prestellar" cores whose densities are above $10^{5} \mathrm{~cm}^{-3}$ and column densities well above $10^{22} \mathrm{~cm}^{-2}$. These cores are probably rather oblivious to what happens around them as they are gravitationally independent entities. Thus, if such cores survived into the bridge region, we would see the star formation occurring.

To be ejected into the bridge region, however, the cores have experienced a sudden deceleration of several $100 \mathrm{~km} \mathrm{~s}^{-1}$. Just as for the rest of the cloud, the collision necessarily ionizes the dense cores as well. However, because the probability of a dense core encountering another dense core is low, the dense core will continue deeper into the incoming cloud, ending up in a lower density medium than the barycenter of the two clouds. It may then be able to expand and lose its "pre-stellar" nature. The ionization and subsequent cooling of the clouds may result in some degree of homogenizing of the clouds, such that time is required before overdensities can become pre-stellar cores.

Is there any evidence for this in the line ratios? Only the Taffy system is strong enough in the CO lines to have any ${ }^{13} \mathrm{CO}$ or HCN observations. Braine et al. (2003) found that the ${ }^{13} \mathrm{CO}$ lines were quite weak in the Taffy bridge, with line ratios of about 50 and 100 respectively in the (1-0) and (2-1) transitions respectively. Taken at face value, these line ratios indicate that the ${ }^{12} \mathrm{CO}$ lines are not as optically thick as they are in galactic clouds or in normal galaxies. In UGC 12915 the ratios are lower, with ratios of 15 and 19 in the (1-0) and (2-1) transitions respectively, close to values in normal spirals. However, in IR-luminous galaxies with very high SFRs, the ${ }^{12 / 13} \mathrm{CO}$ line ratios are also very high (Casoli et al. 1992) and the star formation is particularly efficient. In the IR-luminous galaxies, the $\mathrm{HCN}(1-0)$ line is strong, sometimes stronger than the ${ }^{13} \mathrm{CO}(1-0)$ line (Solomon et al. 1992). In the Taffy bridge, we did not detect the $\mathrm{HCN}(1-0)$ line but the upper limit is only slightly below the ${ }^{13} \mathrm{CO}(1--0)$ line intensity in the bridge. Thus, the line intensities are compatible with a low opacity in the bridge region.

\section{Are head-on collisions cosmologically important?}

It is now generally accepted that galaxies were smaller at high redshift and that local universe galaxies (i.e. with small lookback times and hence current objects rather than early universe objects) are the product of collisions and accretion of smaller entities. The details of this picture remain quite unclear, however, but these collisions are mostly tidal rather than the head-on collisions that are the subject of this work. We now consider two environments, field and dense clusters, to briefly explore whether ISM-ISM collisions could play a role in galaxy evolution for a large number of galaxies.

To keep numbers simple, we will consider spiral galaxies to have a radius of $10 \mathrm{kpc}$ and take a dense cluster environment to have a density of 1000 galaxies per $\mathrm{Mpc}^{3}$ at a velocity of $1000 \mathrm{~km} \mathrm{~s}^{-1}$. The field galaxies are of the same size but have a density of 0.1 galaxies per $\mathrm{Mpc}^{3}$. Going through the same mean free path calculation as before,

$\lambda_{\text {mfp }}=1 /\left(4 A_{\text {gal }} n_{\text {gal }}\right)=1 /\left(4 \pi R^{2} n\right) \approx 800 \mathrm{kpc}$

but at $1000 \mathrm{~km} \mathrm{~s}^{-1}$ a galaxy moves $800 \mathrm{kpc}$ in only $800 \mathrm{Myr}$. In this extreme case, each dense cluster galaxy undergoes an ISM-ISM collision every Gyr! ISM-ISM collisions might then be a very efficient means of bringing gas out of galaxies and into the intracluster medium, especially at earlier epochs when clusters were denser and galaxies more gaseous. The above numbers are probably overestimates: if the galaxies touch at a radius of $10 \mathrm{kpc}$, the collision will not bring gas out of the inner parts - a more appropriate value of $R$ would be $5 \mathrm{kpc}$; furthermore, the galaxies should be assumed to have an average inclination of 45 degrees, reducing the area by $\sqrt{2}$; on the other hand, since the target galaxies move as well, the effective velocity is raised by about the same factor. Nonetheless, each cluster galaxy would still collide every few Gyrs, such that every cluster galaxy would undergo a head-on collision at least once and several times for some objects. Todays rich clusters are spiral-poor but they were certainly much more spiral-rich in the past, as well as being denser. The gas ejected from the inner parts of galaxies would naturally be quite metal-enriched because star formation starts in the inner parts of spirals.

A similar calculation shows that the situation is entirely different for field galaxies. At the much lower density adopted for field spirals, only about one galaxy out of 1000 suffers this sort of collision in 10 Gyr. Even allowing for a density increase of $n(z) \approx n_{0}(1+z)^{3}$, nothing changes out to a redshift of a few at least. We conclude that while these collisions are likely very important for galaxy clusters, they do not affect the evolution of field spirals.

\section{Conclusions}

Consistent with the Taffy galaxies, UGC $12914 / 5$, the second such system, UGC 813/6, also contains large amounts of molecular gas in the bridge region between the galaxies. UGC 813 and 816 are considerably smaller and less gasrich than UGC 12914 and 12915, and the CO emission from the UGC $813 / 6$ bridge is about 4 times less than from the UGC 12914/5 bridge. Nonetheless, the CO emission from the UGC $813 / 6$ bridge is similar to that from the entire Milky Way and the $\mathrm{H}_{2} / \mathrm{HI}$ mass ratio is roughly unity.

It is shown that for reasonably gas-rich, but not extraordinary, spiral galaxies a head-on collision will result in many collisions of molecular clouds. Some earlier calculations had reached the opposite conclusion but they had supposed very 
gas-poor disks. Although the neutral (both atomic and molecular) clouds are ionized in collisions, the dense gas cools very rapidly allowing it to recombine and become molecular while still in the colliding disks. The new cloud is then left in the bridge because the collision has left it with an intermediate velocity.

Some $\mathrm{H}_{2}$ may form through collisions of atomic gas clouds just as some of the originally molecular (or atomic) gas will remain ionized after collision. In addition to showing that GMC-GMC collisions occur in cases where the nuclei of the galaxies have passed within a few kpc of each other, several factors lead us to conclude that GMC-GMC collisions play a major role in bringing molecular gas into the bridge regions. From a theoretical point of view, the study by Hollenbach $\&$ McKee (1989) indicates that a column density of about $4 \times 10^{21} \mathrm{~cm}^{-2}$ is necessary to form $\mathrm{H}_{2}$. This is a large column density for purely atomic clouds. Furthermore, if HI-HI cloud collisions produced the $\mathrm{H}_{2}$, the molecular gas would be as extended as the $\mathrm{HI}$ in the bridge - this is not the case. Being much denser than atomic gas clouds, GMCs will be able to cool much more quickly after collision and ionization, forming the molecular gas we now observe in the bridge.

The star formation in the bridge material is below that expected based on the molecular gas mass. We speculate that the cloud collisions may have eliminated many of the pre-stellar cores, such that in the few $10^{7}$ years since the collision the cores have not had time to reform and generate the strong stellar luminosity expected.

ISM-ISM collisions may provide an efficient mechanism for driving enriched gas out of the inner parts of spirals in galaxy clusters. Given that clusters were even denser in the past, most cluster galaxies have probably undergone such a collision at least once. Only a very small fraction of field spirals, even at high redshift, would have suffered a head-on collision.

The future of the bridge material is as yet unclear. With a velocity of $\$ 300 \mathrm{~km} \mathrm{~s}^{-1}$ with respect to one or another of the spirals, some of the gas is probably destined to fall back onto the parent spirals. Some however may remain in between the receding galaxies.
Acknowledgements. We would like to thank J. J. Condon for the HI and radio continuum data. We would also like to thank Simon Verley of the IAA for taking the $R$ band image used in Figs. 1 and 2 and Raúl Mújica of the INAOE for the $\mathrm{H} \alpha$ image presented in Fig. 4. UL and SL are supported by the Spanish MCyT Grant AYA 2002-03338 and the Junta de Andalucía. VC would like to acknowledge the support of JPL contract 960803.

\section{References}

Bania, T. M., Stark, A. A., \& Heiligman, G. M. 1986, ApJ, 307, 350

Braine, J., Davoust, E., Zhu, M., et al. 2003, A\&A, 408, L13

Braine, J., Duc, P.-A., Lisenfeld, U., et al. 2001, A\&A, 378, 51

Bushouse, H. A. 1987, ApJ, 320, 49

Bushouse, H. A., \& Werner, M. W. 1990, ApJ, 359, 72

Casoli, F., Dupraz, C., \& Combes, F. 1992, A\&A, 264, 55

Condon, J. J., Helou, G., \& Jarrett, T. H. 2002, AJ, 123, 1881

Condon, J. J., Helou, G., Sanders, D. B., \& Soifer, B. T. 1993, AJ, 105,1730

Crutcher, R. M. 1999, ApJ, 520, 706

Dickman, R. L., Snell, R. L., \& Schloerb, F. P. 1986, ApJ, 309, 326

Gaetz, T. J., \& Salpeter, E. E. 1983, ApJS, 52, 155

Gao, Y., Zhu, M., \& Seaquist, E. 2003, ApJ, in press

Harwit, M., Houck, J. R., Soifer, B. T., \& Palumbo, G. G. C. 1987, ApJ, 315, 28

Hollenbach, D., \& McKee, C. F. 1989, ApJ, 342, 306

Hollenbach, D., \& Salpeter, E. E. 1971, ApJ, 163, 155

Jarrett, T. H., Helou, G., Van Buren, D., Valjavec, E., \& Condon, J. J. 1999, AJ, 118, 2132

Jog, C. J., \& Solomon, P. M. 1992, ApJ, 387, 152

Kennicutt, R. C. 1998, ApJ, 498, 541

Larson, R. B. 1981, MNRAS, 194, 809

Mair, G., Mueller, E., Hillebrandt, W., \& Arnold, C. N. 1988, A\&A, 199, 114

Solomon, P. M., Downes, D., \& Radford, S. J. E. 1992, ApJ, 387, L55

Struck, C. 1997, ApJS, 113, 269

Zhu, M., Seaquist, E. R., Davoust, E., Frayer, D. T., \& Bushouse, H. A. 1999, AJ, 118, 145

Zink, E. C., Lester, D. F., Doppmann, G., \& Harvey, P. M. 2000, ApJS, 131,413 